\title{
An image fusion system for corrective osteotomy of distal radius malunion
}

\author{
Yuichi Yoshii ${ }^{*} \mathbb{B}^{0}$, Takeshi Ogawa², Yuki Hara², Yasukazu Totoki² and Tomoo Ishii ${ }^{1}$
}

\section{*Correspondence:}

yyoshii@tokyo-med.ac.jp

1 Department of Orthopaedic

Surgery, Tokyo Medical

University Ibaraki Medical

Center, 3-20-1 Chuo, Ami,

Inashiki, Ibaraki 300-0395,

Japan

Full list of author information

is available at the end of the article

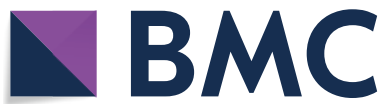

(c) The Author(s) 2021. This article is licensed under a Creative Commons Attribution 4.0 International License, which permits use, sharing, adaptation, distribution and reproduction in any medium or format, as long as you give appropriate credit to the original author(s) and the source, provide a link to the Creative Commons licence, and indicate if changes were made. The images or other third party material in this article are included in the article's Creative Commons licence, unless indicated otherwise in a credit line to the material. If material is not included in the article's Creative Commons licence and your intended use is not permitted by statutory regulation or exceeds the permitted use, you will need to obtain permission directly from the copyright holder. To view a copy of this licence, visit http://creativecommons.org/ licenses/by/4.0/. The Creative Commons Public Domain Dedication waiver (http://creativecommons.org/publicdomain/zero/1.0/) applies to the data made available in this article, unless otherwise stated in a credit line to the data. 


\section{Background}

Distal radius malunion is a common complication of distal radius fractures. It occurs when a fracture of the distal radius heals with improper alignment, incorrect length, articular incongruity, or a combination of these factors. The incidence of distal radius malunion was previously reported to be approximately 5-24\% of distal radius fractures [1-4]. Most cases of distal radius malunion are symptomatic due to changes in biomechanical conditions. It causes pain, weakness, or functional impairment at the wrist joint [1,5-7]. Symptomatic distal radius malunion requires corrective osteotomy. The aim of corrective osteotomy is to reduce pain and improve wrist function. It needs to restore normal wrist joint congruency and realign the distal radius.

Preoperative planning for corrective osteotomy has traditionally been performed using two-dimensional (2D) radiographs of the anterior-posterior and lateral views [8-10]. However, conventional X-rays cannot sufficiently evaluate rotational deformities [9, 11, 12]. The outcomes of corrective osteotomy for rotational corrections using $2 \mathrm{D}$ imaging are mostly unsatisfactory [11]. In recent years, three-dimensional (3D) planning, navigation, and the possibility of implant customization have led to changes in the clinical practice of corrective osteotomy for distal radius malunion. 3D preoperative planning visualizes the reduction process and placement/choices of implants. It is particularly useful for visualizing the correction of rotational deformities [10, 13]. These innovations have been possible due to advances in the field of computer-assisted technologies. Although significant advances have been made in 3D visualization and planning, the use of $2 \mathrm{D}$ to $3 \mathrm{D}$ or $3 \mathrm{D}$ to $2 \mathrm{D}$ image conversion in clinical practice has not yet been perfected. To more smoothly perform 2D-3D conversion for osteosynthesis and osteotomy, we developed an image fusion system for 3D preoperative planning and fluoroscopy. This system draws an outline of a 3D preoperative planning image and projects it onto a fluoroscopic image [14]. To assess the utility of the image fusion system, we evaluated the reproducibility of preoperative planning in corrective osteotomy for dorsally angulated distal radius malunion using the system. The results obtained were compared to the reproducibility of a preoperative plan without using the system.

\section{Results}

The results of correction accuracy for the reference points are shown in Fig. 1. The distances between preoperative planning and postoperative reduction in the image fusion group were $2.1 \pm 1.1 \mathrm{~mm}, 1.8 \pm 0.7 \mathrm{~mm}, 1.9 \pm 0.9 \mathrm{~mm}$, and $1.4 \pm 0.7 \mathrm{~mm}$ for reference points (1), (2), (3), and the barycenter, respectively. The distances between preoperative planning and postoperative reduction in the control group were $3.7 \pm 1.0 \mathrm{~mm}$, $2.8 \pm 2.0 \mathrm{~mm}, 1.7 \pm 0.8 \mathrm{~mm}$, and $1.8 \pm 1.2 \mathrm{~mm}$ for reference points (1), (2), (3), and the barycenter, respectively. The difference in reference point (1) was significantly smaller in the image fusion group than in the control group $(\mathrm{P}<0.05)$.

The results of correction accuracy for radial inclination and volar tilt on $3 \mathrm{D}$ images (3DRI, 3DVT) are shown in Fig. 2. Differences between the preoperative and postoperative plans in the image fusion group were $3.0 \pm 0.9^{\circ}$ and $2.5 \pm 0.6^{\circ}$ for $3 \mathrm{DRI}$ and $3 \mathrm{DVT}$, respectively, while those in the control group were $2.7 \pm 3.2^{\circ}$ and $5.5 \pm 3.5^{\circ}$ for $3 \mathrm{DRI}$ and 3DVT, respectively. Small differences in 3DVT were observed in the image fusion group 

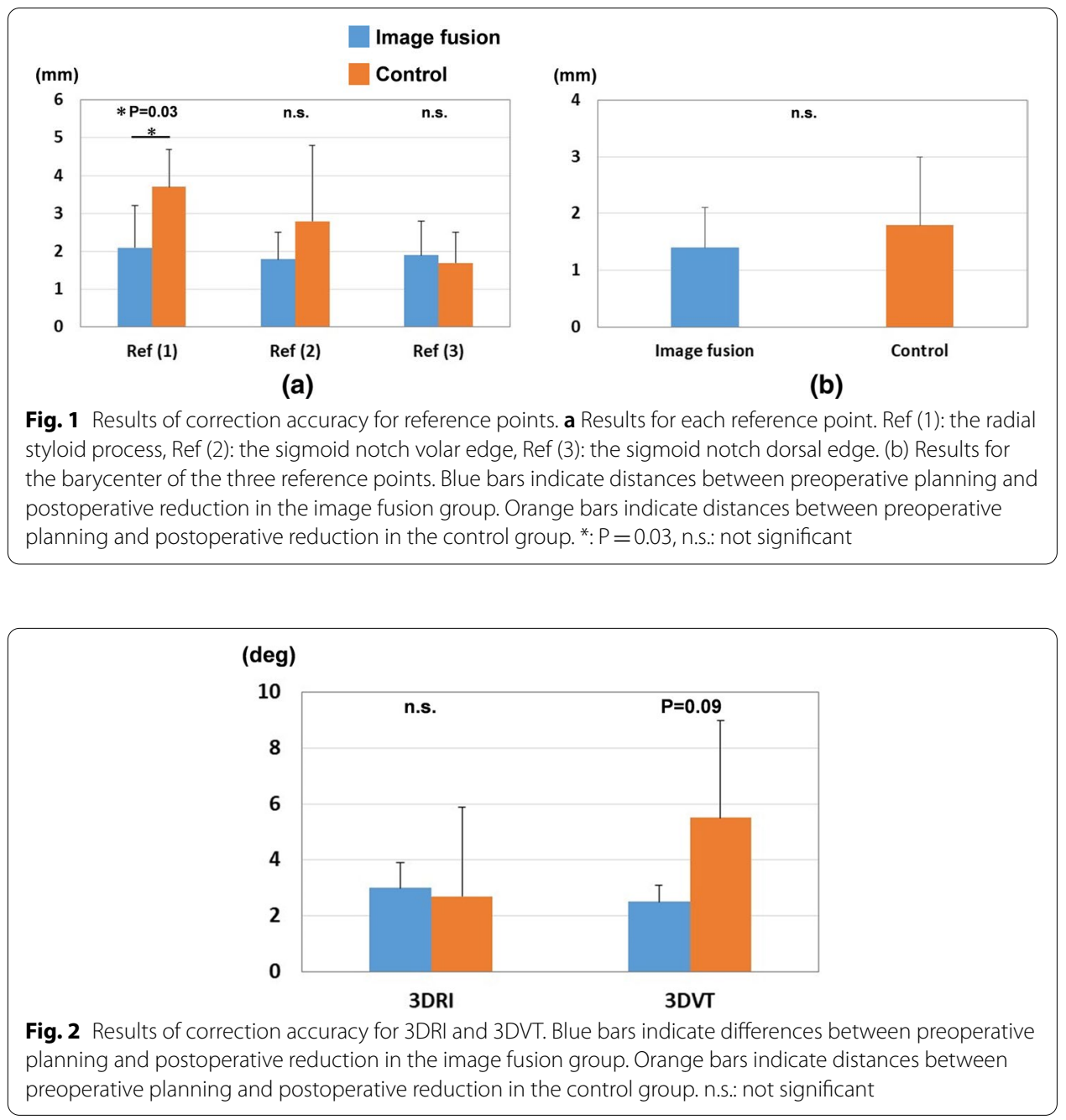

$(\mathrm{P}=0.09)$. Mayo wrist scores were 52.5/58.8 and 78.8/68.8 (image fusion group / control group) in three and six months after surgery, respectively.

\section{Discussion}

The present study evaluated the utility of our image fusion system in corrective osteotomy for distal radius malunion. A 3D simulation in combination with a fluoroscopic image is a novel surgical approach for corrective osteotomy. The image fusion system allows us to directly compare preoperative planning images with fluoroscopy images. With a visual guide during the surgical process, the surgeon had more confidence regarding implant placement and osteotomy. As a result, the reproducibility of the 3D preoperative plan for corrective osteotomy improved.

Since it is difficult to carry out a corrective osteotomy plan during actual surgery, several computer-aided techniques have been introduced [13]. With the spread of analytical software and 3D printers, the 3D visualization of osteotomy and preoperative evaluations are becoming more common [15]. Previous studies reported the use of optical tracking devices and synthetic templates to reproduce reduction shapes [16-19]. More recently, the possibility of computer assistance for corrective osteotomy by creating 
patient-specific surgical cutting guides and fixation plates was reported [20,21]. The use of patient-specific guides is currently considered to be the most accurate method, but involves extra costs to perform surgery. Furthermore, positioning errors in the patientspecific guide affect correction accuracy [22]. The image fusion system allows the 3D preoperative plan to be directly compared with the fluoroscopic image without additional medical expenses. This is one of the benefits of using the image fusion system.

The reduction position of each reference point was reproducible with an error of 1.9$2.1 \mathrm{~mm}$ using the image fusion system. Previous studies showed a reduction accuracy of less than $2 \mathrm{~mm}$ when computer-aided technology was used in osteotomy for distal radius malunion [23-25]. Patient-specific instruments were used in these studies. Other studies demonstrated that in conventional osteotomy, only $40 \%$ of corrections achieved within 5 degrees of the planned correction for the angular deformity and within $2 \mathrm{~mm}$ of the planned ulnar variance [26]. The image fusion system does not require specific instruments. Nevertheless, the reduction accuracy of each reference point was similar to that obtained using patient-specific instruments. The difference between preoperative plan and postoperative reduction for the reference point (1) was slightly larger than other reference points because distal radius malunion is mostly deformed in the dorsal, radial and proximal directions. Therefore, the amount of movement for the radial styloid process becomes larger than other reference points. This difference tended to increase if the reduction could not be achieved accurately. Considering the improved reproducibility of reference point (1) and 3DVT, this may represent a strategy for improving reduction accuracy without using patient-specific instruments. Therefore, we propose this approach as a novel method for performing corrective osteotomy.

There were several limitations that need to be addressed. This was a case control study without randomization. The control group was enrolled from previous cases. Randomized controlled trials may be difficult to conduct due to the limited number of cases in a single facility. Large-scale investigations, such as case registration, may be needed to evaluate true practicality. Although image fusion in clinical medicine is already a well-known technology, there are two novelties in the present study: the use of an image fusion method in corrective osteotomy and the conversion of a 3D preoperative plan into a $2 \mathrm{D}$ contour extraction image, thereby allowing existing fluoroscopy to be used to perform image fusion. Since this is a preliminary study, the number of cases was limited. In this study, we attempted to establish a protocol for corrective osteotomy using the image fusion system. If this method is accepted, we plan to adapt it to more cases and verify its clinical significance. Another limitation is that the implementation of this method requires specialized software and proficiency in the procedure. There may be a learning curve and improved accuracy following the mastery of the procedure. This method also involves CT scans. In the present study, axial CT images with a slice thickness of $1-1.5 \mathrm{~mm}$ were used. Radiation exposure needs to be reduced by increasing the slice thickness or changing imaging conditions. Furthermore, no significant differences were observed in clinical outcomes. In a previous meta-analysis, 3D-planned corrective osteotomy significantly improved both radiographical and functional outcomes over the preoperative condition. However, it is still unclear whether these computer-assisted technologies improve clinical outcomes. This method may help residents and fellows who are unfamiliar with corrective osteotomy to visualize surgery and share information 
with other medical staff. In addition, the evaluation method presented herein may be applied to the biomechanical analysis of distal radius fractures. These educational and academic significances need to be investigated in future studies.

\section{Conclusions}

An image fusion system was developed for corrective osteotomy. 3D reference points were reproducible with an error of approximately $2 \mathrm{~mm}$ in corrective osteotomy for distal radius malunion using the system. The reproducibility of the 3D preoperative plan for corrective osteotomy was improved using the image fusion system. This image fusion system represents an approach to reproduce the planned reduction in corrective osteotomy for distal radius fracture malunion.

\section{Methods}

The study protocol was approved by the Institutional Review Board (No. 18-19, T20190178). This was a retrospective comparative study (Level of Evidence III). It has been registered as NCT03764501 at ClinicalTrials.gov (registered 21 May, 2018, retrospectively registered). This study was performed in accordance with the relevant guidelines and regulations. Informed consent was obtained from all individual participants included in the study. Ten wrists from 10 distal radius malunion patients who underwent corrective osteotomy (six females, four males, mean age 59.4 years, age range 40-78) were evaluated. The image fusion group underwent 3D preoperative planning and performed corrective osteotomy with an image fusion system $(n=5)$. The control group was enrolled from patients who underwent corrective osteotomy using only 3D preoperative planning $(\mathrm{n}=5)$.

\section{Preoperative planning}

In both groups, 3D digital preoperative planning and a surgical simulation were performed prior to surgery. Corrections to and the placement of implants were simulated using software developed by one of the authors (Zed-Trauma, LEXI Co., Ltd., Tokyo, Japan). Computed tomography (CT) of the affected and unaffected wrists using contiguous images with a slice thickness of 1-1.5 mm were taken for the simulation. Images were taken approximately $12 \mathrm{~cm}$ proximal to the radial joint surface. A total of $80-120$ axial CT images were used for the simulation. After importing DICOM images into the software, a 3D image of the distal radius was made for both wrists. In dorsally angulated malunion, the placement of a volar locking plate was initially simulated. Computeraided design models of different-sized implants are installed in the software; a placement image of the plate was created by calculating the correction angle required to restore volar tilt, radial inclination, and rotational deformation (Fig. 3). Stellar II locking plates (HOYA Technosurgical, Inc., Tokyo, Japan) were used in the present study. This plate system has small, medium, and large widths as well as short and long plate lengths. The plate size was selected to cover the distal radius maximally and not exceed the width of the distal radius. In addition, a sufficient length plate for the insertion of at least three screws into the radius shaft after reduction was chosen. The lengths for the distal screws were selected and a contour extraction image of the initial plate placement was saved for image fusion. The osteotomy line was set at a position that did not interfere with 


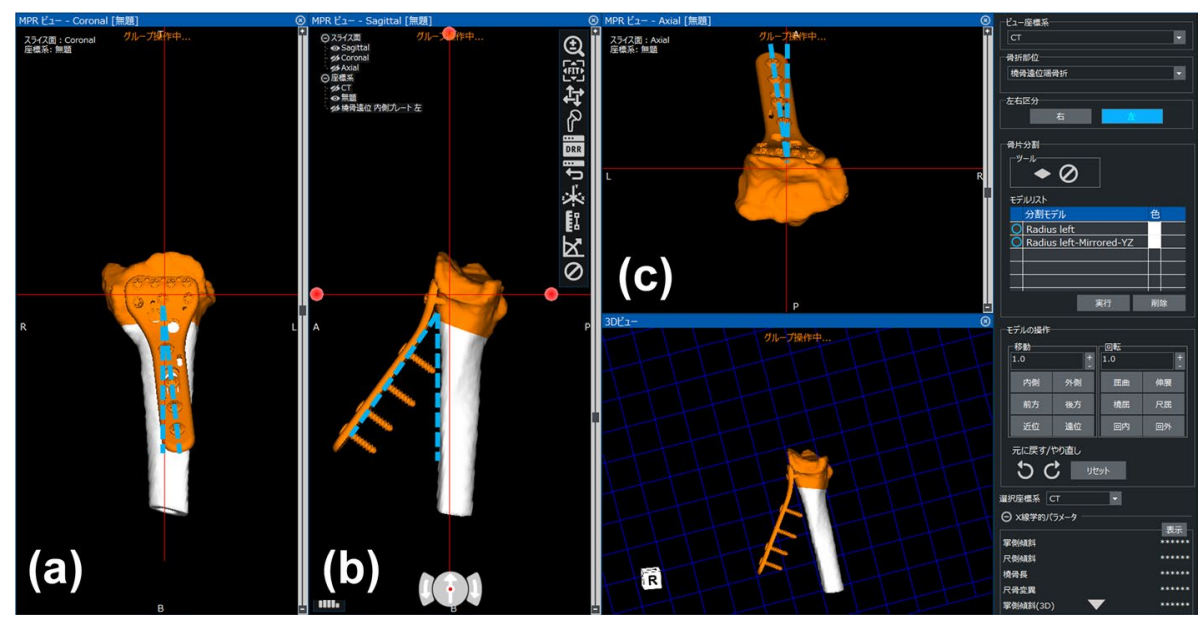

Fig. 3 Preoperative image of plate placement. An image of plate placement was created by calculating the correction angle required to restore volar tilt, radial inclination, and rotational deformation. The plate was placed so that the distal margin was parallel to the articular surface. An image of each frame shows the plate fixed to the distal radius. The blue dotted line on the frame (a) shows the correction angles for the coronal view. The blue dotted line on the frame (b) shows the correction angles for the sagittal view. The blue dotted line on the frame (c) shows the correction angles for the axial view

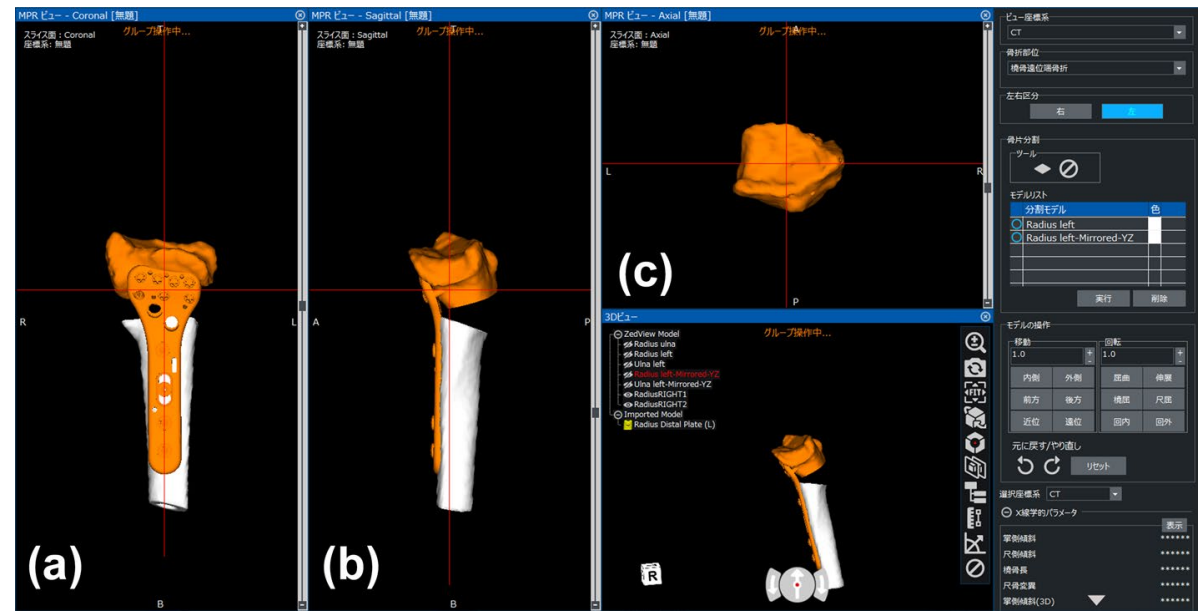

Fig. 4 Preoperative image of reduction. After separating the distal part of the radius, reduction was achieved by fixing the proximal side of the plate to the radius shaft. The gap between the distal and proximal parts of the radius indicates the predicted bone defect. a Coronal view, $\mathbf{b}$ sagittal view, c axial view

distal screw holes. After the osteotomy simulation, the plate and distal fragment were grouped, and the distal fragment was repositioned by adapting the proximal side of the volar locking plate to the radius shaft (Fig. 4). After repositioning the fragments, the 3D bone shape was compared with a mirror image of the unaffected radius. In the next step, simulations of the screw choices were performed for the proximal screw holes and the screw lengths for each screw hole were selected. This final reduction and implant placement image was also saved for image fusion. To compare planned and postoperative reduction shapes, preoperative plan and postoperative 3D images were created for all patients. 


\section{Image fusion system and surgical intervention}

Regarding image fusion, 3D images of preoperative plans were converted to digitally reconstructed radiographs. Bone and implant contour extraction images were created for anterior-posterior and lateral views based on 3D images. Fusion images were displayed on a monitor overlapping the outline of the 3D preoperative plan and the fluoroscopic image. Corrective osteotomy was performed under general anesthesia. In the image fusion group, the outline of the planned image was displayed on a monitor overlapping the fluoroscopy image during surgery. Before starting surgery, the contour extraction image size was calibrated by measuring a known length. A surgeon performed corrective osteotomy based on the fusion image. Before osteotomy, a plate placement image was displayed on the monitor (Fig. 5). In the first step, the plate was placed on the distal radius according to the outline of the plate image and fixed with two temporary fixing wires. Outlines of the anterior-posterior and lateral views were used. According to the direction of the fluoroscopic image, the direction of the contour image was changed to the anterior-posterior or lateral view. Plate placement was checked with a fusion image for each direction. After determining the plate position at the distal radius, distal screw holes were pre-drilled. The plate was then removed leaving the temporary fixing wires. In the second step, osteotomy was performed at a level that did not interfere with the distal screws of the plate. In the third step, the plate was returned to the originally selected position under the guide of the temporary fixing wires, and the distal screws were inserted into the pre-drilled holes. Finally, the distal fragment was repositioned by adapting the proximal side of the volar locking plate to the radius shaft, and the plate was fixed with screws (Fig. 6).

In the control group, the surgeon performed reduction and placement of the plate while comparing separate images of the 3D preoperative plan and fluoroscopy during surgery. In both groups, a beta-tricalcium phosphate-based artificial bone or autologous bone graft was performed depending on the size of the bone defect. Surgeries were performed by one hand surgeon.

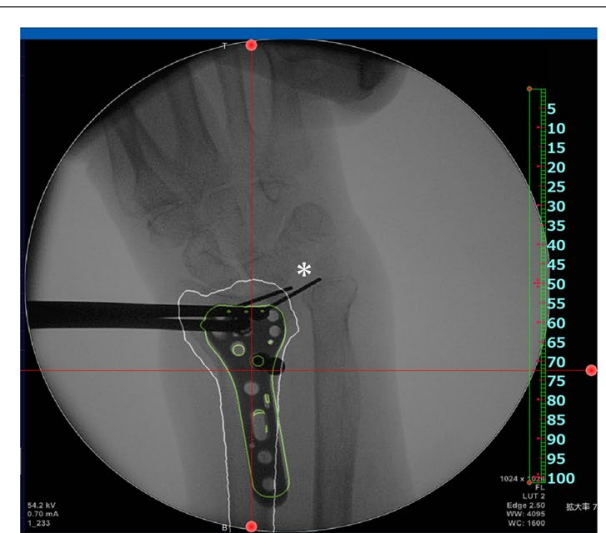

(a)

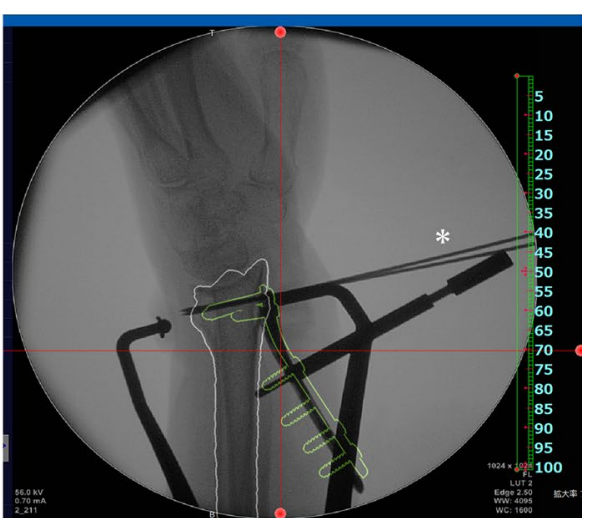

(b)

Fig. 5 Fusion image of the preoperative plan and fluoroscopy for plate placement. Based on the plate placement image, a contour extraction image was created and displayed on the fluoroscopy image for surgery. The plate was placed to fit the contour line. a Anterior-posterior view, b lateral view. ${ }^{*}$ shows temporary fixing wires 


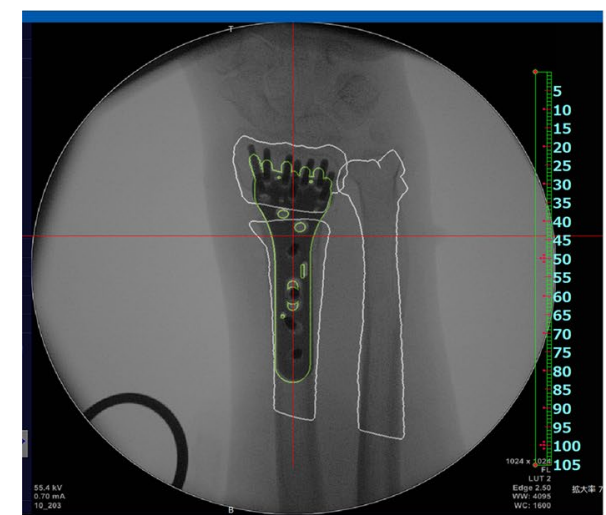

(a)

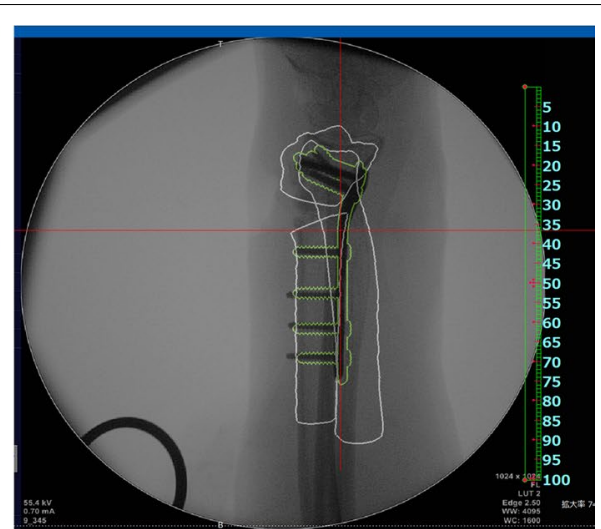

(b)

Fig. 6 Fusion image of the preoperative plan and fluoroscopy for reduction. Based on the reduction image, the distal fragment was repositioned by adapting the proximal side of the volar locking plate to the contour extraction image

\section{Evaluations}

Pre- and post-operative 3D images of the distal radius were analyzed using image analysis software (BoneSimulater, Orthree, Osaka, Japan). DICOM data of CT images were imported into the software. A 3D surface model of the radius was constructed with a surface construction algorithm. The long axis of the radius was calculated from the 3D surface model of the intact part of the preoperative distal radius image. Image registration for the preoperative plan and postoperative reduction were performed using the intact part of the distal radius image. The y-axis was defined as the long axis of the radius, and the proximal direction was defined as positive. The $\mathrm{z}$-axis was parallel to the orthogonal projection of a line initiating at the base of the distal ulnar sigmoid notch and continuing to the radial styloid process on a plane perpendicular to the $y$-axis. The radial direction on the $\mathrm{z}$-axis was defined as positive. The $\mathrm{x}$-axis was normal to the yz plane and the palmar direction was defined as positive. The yz, $\mathrm{xy}$, and $\mathrm{xz}$ planes were defined as the coronal, sagittal, and axial planes, respectively. The origin of coordinates was defined as the intersection of the joint surface and the radius of the long axis on the preoperative plan image. Three reference points: (1) the radial styloid process, (2) the sigmoid notch volar edge, and (3) the sigmoid notch dorsal edge, were marked on pre- and post-operative 3D images (Fig. 7). The 3D coordinates of each reference point and the barycentric coordinates of the plane connecting the three reference points were evaluated using the 3D images of the preoperative plan and postoperative reduction.

In the sagittal view, the angle between a connecting line from reference point (2) to reference point (3) and a line perpendicular to the longitudinal axis of the radius was measured as volar tilt (3DVT). In the coronal view, the angle between a line from reference point (1) to reference point (2) and a line perpendicular to the longitudinal axis of the radius was measured as radial inclination (3DRI).

In evaluations of clinical outcomes, Mayo wrist scores [27] were recorded 3 and 6 months after surgery. 

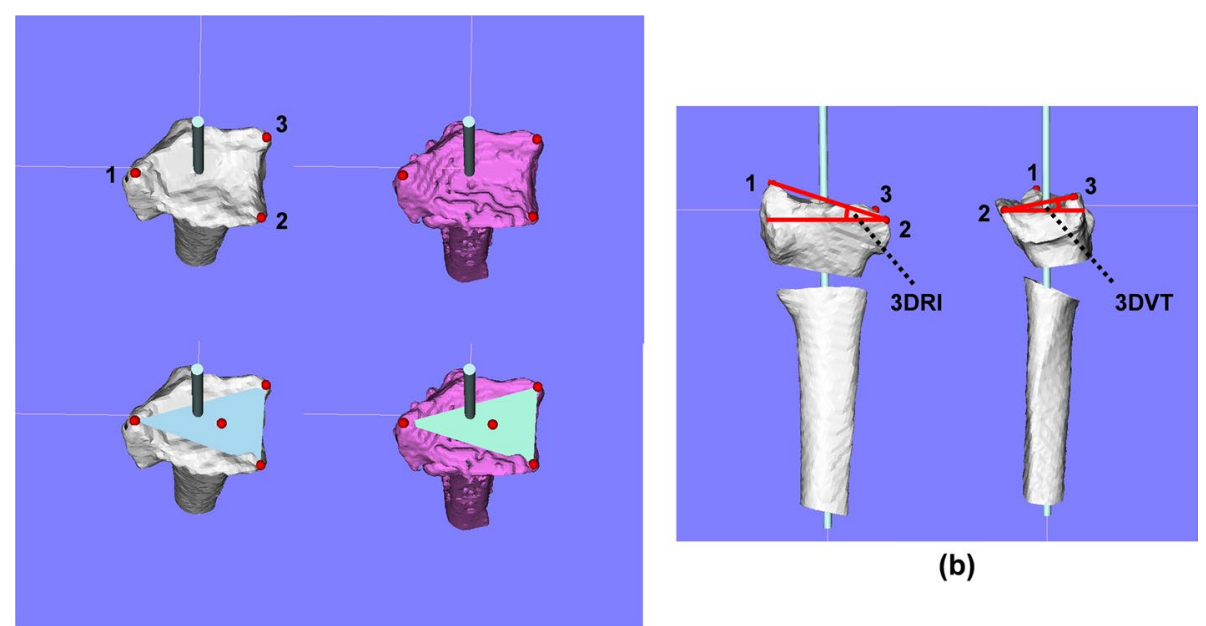

(b)

(a)

Fig. 7 Example images of 3D reference points. a An example image for each reference point. Left row images show the reference points in the preoperative plan image. Right row images show the reference points in the postoperative image. The light blue bar indicates the long axis of the distal radius. Red dots indicate the radial styloid process: reference point (1), the sigmoid notch volar edge: reference point (2), the sigmoid notch dorsal edge: reference point (3), and the barycentric coordinates. b An example image for 3DVT and 3DRI. The angle between a connecting line from reference point (2) to reference point (3) and a line perpendicular to the longitudinal axis of the radius was measured as the volar tilt on a 3D image in the sagittal view (3DVT). The angle between a line from reference point (1) to reference point (2) and a line perpendicular to the longitudinal axis of the radius was measured as the radial inclination on a 3D image in the coronal view (3DRI). The measured angles in the example image were $19.2^{\circ}$ and $12.7^{\circ}$ for $3 \mathrm{DRI}$ and $3 \mathrm{DVVT}$, respectively

\section{Statistical analysis}

Results are expressed as the mean \pm standard deviation. Distances between the preoperative plan and postoperative reduction for each reference point were measured for both groups. Differences between the preoperative plan and postoperative reduction for 3DRI and 3DVT were measured for both groups. The Shapiro-Wilk test was used to test the normality of datasets. The distances of reference points and differences in angles were compared using Welch's $t$-test. The Mann-Whitney U test was used for unevenly distributed datasets (3DVT). P values of less than 0.05 were considered to be significant. All analyses were performed using BellCurve for Excel version 2.12 (SSRI Co., Tokyo, Japan).

\section{Abbreviations}

3D: Three-dimensional; 2D: Two-dimensional; DICOM: Digital imaging and communications in medicine; CT: Computed tomography; 3DVT: Three-dimensional volar tilt; 3DRI: Three-dimensional radial inclination.

\section{Acknowledgements}

Not applicable.

\section{Authors' contributions}

YY: research design, analysis of data, wrote the manuscript. TO: acquisition and analysis of data, wrote the manuscript. $\mathrm{YH}$ : acquisition and analysis of data, wrote the manuscript. YT: acquisition and analysis of data. Tl: analysis of data, wrote the manuscript, supervised the study. All authors were fully involved in the study. All authors read and approved the final manuscript.

\section{Funding}

The present study was supported by a Grant-in-Aid for Scientific Research (19K09582) and AMED (A324TS). These funds did not affect data collection, data analysis, or the preparation or editing of the manuscript. 
Availability of data and materials

The datasets used and/or analyzed during the present study are available from the corresponding author upon reasonable request.

\section{Declarations}

\section{Ethics approval and consent to participate}

The protocol for the present study was reviewed and approved by the Institutional Review Board of Tokyo Medical University Ibaraki Medical Center (No. 14-21, T2019-0178). This study has been registered as NCT03764501 at ClinicalTrials. gov. We followed the CONSORT guidelines to perform this study.

\section{Consent for publication}

Written consent for publication was obtained from all study participants.

\section{Competing interests}

No benefits in any form have been received or will be received from a commercial party related directly or indirectly to the subject of this article. The image fusion system described in the present study has been developed through a collaborative study between Tokyo Medical University Ibaraki Medical Center and LEXI Co., Ltd.

\section{Author details}

${ }^{1}$ Department of Orthopaedic Surgery, Tokyo Medical University Ibaraki Medical Center, 3-20-1 Chuo, Ami, Inashiki, Ibaraki 300-0395, Japan. ${ }^{2}$ Department of Orthopaedic Surgery, University of Tsukuba Hospital, Tsukuba, Ibaraki 305-8576, Japan.

Received: 14 December 2020 Accepted: 20 June 2021

Published online: 30 June 2021

\section{References}

1. Cooney WP, Dobyns JH, Linscheid RL. Complications of Colles' fractures. J Bone Joint Surg Am. 1980;62:613-9.

2. Mulders MAM, d'Ailly PN, Cleffken BI, Schep NWL. Corrective osteotomy is an effective method of treating distal radius malunions with good long-term functional results. Injury. 2017:48:731-7.

3. Viegas SF. A new modification of corrective osteotomy for treatment of distal radius malunion. Tech Hand Uppe Extremity Surg. 2006;10:224-30.

4. Katt B, Seigerman D, Lutsky K, Beredjiklian P. Distal radius malunion. J Hand Surg Am. 2020;45:433-42.

5. Bushnell BD, Bynum DK. Malunion of the distal radius. J Am Acad Orthop Surg. 2007;15:27-40.

6. Graham TJ. Surgical correction of malunited fractures of the distal radius. J Am Acad Orthop Surg. 1997:5:270-81.

7. Park MJ, Cooney WP, Hahn ME, Looi KP, An KN. The effects of dorsally angulated distal radius fractures on carpal kinematics. J Hand Surg Am. 2002;27:223-32.

8. Dobbe JGG, Vroemen JC, Strackee SD, Streekstra GJ. Corrective distal radius osteotomy: including bilateral differences in 3-D planning. Med Biol Eng Comput. 2013;51:791-7.

9. Ring D, Prommersberger K, Del Pino J, Capomassi M, Slullitel M, Jupiter JB. Corrective osteotomy for intra-articular malunion of the distal part of the radius. J Bone Joint Surg Am. 2005;87:1503-9.

10. Vroemen JC, Dobbe JGG, Sierevelt IN, Strackee SD, Streekstra GJ. Accuracy of distal radius positioning using an anatomical plate. Orthopedics. 2013;36:457-62.

11. Cirpar M, Gudemez E, Cetik O, Turker M, Eksioglu F. Rotational deformity affects radiographic measurements in distal radius malunion. Eur J Orthop Surg Traumatol. 2011;21:13-20. https://www.researchgate.net/publication/22545 2320.

12. Vroemen JC, Dobbe JGG, Strackee SD, Streekstra GJ. Positioning evaluation of corrective osteotomy for the malunited radius: 3-D CT versus 2-D radiographs. Orthopedics. 2013;36:e193-9.

13. de Muinck Keizer RJO, Lechner KM, Mulders MAM, Schep NWL, Eygendaal D, Goslings JC. Three-dimensional virtual planning of corrective osteotomies of distal radius malunions: a systematic review and meta-analysis. Strategies Trauma Limb Reconstr. 2017;12:77-89.

14. Yoshii Y, Totoki Y, Sashida S, Sakai S, Ishii T. Utility of an image fusion system for 3D preoperative planning and fluoroscopy in the osteosynthesis of distal radius fractures. J Orthop Surg Res. 2019;14:342.

15. Walenkamp MMJ, de Muinck Keizer RJO, Dobbe JGG, Streekstra GJ, Goslings JC, Kloen P, Strackee SD, Schep NWL. Computer-assisted 3D planned corrective osteotomies in eight malunited radius fractures. Strateg Trauma Limb Reconstr. 2015;10:109-16.

16. Athwal GS, Ellis RE, Small CF, Pichora DR. Computer-assisted distal radius osteotomy. J Hand Surg Am. 2003;28:951-8.

17. Croitoru H, Ellis RE, Prihar R. Fixation-based surgery: a new technique for distal radius osteotomy. Comput Aided Surg. 2001;6:160-9.

18. Rieger M, Gabl M, Gruber H, Jaschke WR, Mallouhi A. CT virtual reality in the preoperative workup of malunited distal radius fractures: preliminary results. Eur Radiol. 2005;15:792-7.

19. Honigmann P, Thieringer F, Steiger R, Haefeli M, Schumacher R, Henning J. A simple 3-dimensional-printed aid for a corrective palmar opening wedge osteotomy of the distal radius. J Hand Surg Am. 2016;41:464-9.

20. Murase T, Oka K, Moritomo H, Goto A, Yoshikawa H, Sugamoto K. Three-dimensional corrective osteotomy of malunited fractures of the upper extremity with use of a computer simulation system. J Bone Joint Surg Am. 2008:90:2375-89. 
21. Oka K, Moritomo H, Goto A, Sugamoto K, Yoshikawa H, Murase T. Corrective osteotomy for malunited intra-articular fracture of the distal radius using a custom-made surgical guide based on three-dimensional computer simulation: case report. J Hand Surg Am. 2008;33:835-40.

22. Caiti G, Dobbe JGG, Strijkers GJ, Strackee SD, Streekstra1 GJ. Positioning error of custom 3D-printed surgical guides for the radius: influence of fitting location and guide design. Int J Comput Assist Radiol Surg. 2018;13:507-18.

23. Omori S, Murase T, Kataoka T, Kawanishi Y, Oura K, Miyake J, Tanaka H, Yoshikawa H. Three-dimensional corrective osteotomy using a patient-specific osteotomy guide and bone plate based on a computer simulation system: accuracy analysis in a cadaver study. Int J Med Robot. 2014;10:196-202.

24. Roner S, Vlachopoulos L, Nagy L, Schweizer A, Fürnstahl P. Accuracy and early clinical outcome of 3-dimensional planned and guided single-cut osteotomies of malunited forearm bones. J Hand Surg Am. 2017;42:1031.e1-e8.

25. Stockmans F, Dezillie M, Vanhaecke J. Accuracy of 3D virtual planning of corrective osteotomies of the distal radius. J Wrist Surg. 2013;2:306-14.

26. von Campe A, Nagy L, Arbab D, Dumont CE. Corrective osteotomies in malunions of the distal radius: do we get what we planned? Clin Orthop Relat Res. 2006;450:179-85.

27. Amadio PC, Berquist TH, Smith DK, Ilstrup DM, Cooney WP, Linscheid RL. Scaphoid malunion. J Hand Surg Am. 1989:14:679-87.

\section{Publisher's Note}

Springer Nature remains neutral with regard to jurisdictional claims in published maps and institutional affiliations.

- fast, convenient online submission

- thorough peer review by experienced researchers in your field

- rapid publication on acceptance

- support for research data, including large and complex data types

- gold Open Access which fosters wider collaboration and increased citations

- maximum visibility for your research: over $100 \mathrm{M}$ website views per year

At BMC, research is always in progress.

Learn more biomedcentral.com/submissions 